\title{
Anesthesia considerations to reduce motion and atelectasis during advanced guided bronchoscopy
}

\author{
Michael A. Pritchett ${ }^{1 *}$, Kelvin Lau ${ }^{2}$, Scott Skibo ${ }^{3}$, Karen A. Phillips ${ }^{4}$ and Krish Bhadra ${ }^{5}$
}

\begin{abstract}
Partnership between anesthesia providers and proceduralists is essential to ensure patient safety and optimize outcomes. A renewed importance of this axiom has emerged in advanced bronchoscopy and interventional pulmonology. While anesthesia-induced atelectasis is common, it is not typically clinically significant. Advanced guided bronchoscopic biopsy is an exception in which anesthesia protocols substantially impact outcomes. Procedure success depends on careful ventilation to avoid excessive motion, reduce distortion causing computed tomography (CT)-to-body-divergence, stabilize dependent areas, and optimize breath-hold maneuvers to prevent atelectasis. Herein are anesthesia recommendations during guided bronchoscopy. $\mathrm{An} \mathrm{FiO}_{2}$ of 0.6 to 0.8 is recommended for preoxygenation, maintained at the lowest tolerable level for the entire the procedure. Expeditious intubation (not rapidsequence) with a larger endotracheal tube and non-depolarizing muscle relaxants are preferred. Positive end-expiratory pressure (PEEP) of up to $10-12 \mathrm{~cm} \mathrm{H}_{2} \mathrm{O}$ and increased tidal volumes help to maintain optimal lung inflation, if tolerated by the patient as determined during recruitment. A breath-hold is required to reduce motion artifact during intraprocedural imaging (e.g., cone-beam CT, digital tomosynthesis), timed at the end of a normal tidal breath (peak inspiration) and held until pressures equilibrate and the imaging cycle is complete. Use of the adjustable pressurelimiting valve is critical to maintain the desired PEEP and reduce movement during breath-hold maneuvers. These measures will reduce atelectasis and CT-to-body divergence, minimize motion artifact, and provide clearer, more accurate images during guided bronchoscopy. Following these recommendations will facilitate a successful lung biopsy, potentially accelerating the time to treatment by avoiding additional biopsies. Application of these methods should be at the discretion of the anesthesiologist and the proceduralist; best medical judgement should be used in all cases to ensure the safety of the patient.
\end{abstract}

Keywords: Atelectasis, General anesthesia, Electromagnetic navigation bronchoscopy, Radial endobronchial ultrasound, Image-guided bronchoscopy, Computed tomography, Divergence, Lung cancer

\section{Background}

Advanced guided bronchoscopic biopsy is a minimally invasive method to diagnose suspicious lung lesions [1, 2]. Guidance modalities include not only intraprocedural

\footnotetext{
*Correspondence: mpritchett@pinehurstmedical.com

${ }^{1}$ Chest Center of the Carolinas at First Health, President of the Society for Advanced Bronchoscopy, FirstHealth of the Carolinas and Pinehurst Medical Clinic, 205 Page Road, Pinehurst, NC 28374, USA

Full list of author information is available at the end of the article
}

image-guided methods (e.g., augmented fluoroscopy, tomosynthesis-based fluoroscopic navigation), but also electromagnetic navigation bronchoscopy (ENB), electromagnetic-based and fiber-optic shape-sensing robotic bronchoscopy, virtual bronchoscopic navigation (VBN), radial endobronchial ultrasound (EBUS), and cone-beam computed tomography (CBCT) $[1,2]$. Guided bronchoscopy has a lower risk of complications than transthoracic biopsy [3, 4], and is particularly useful for small lesions in 
the periphery of the lung that cannot be reached by traditional bronchoscopy [5]. Early diagnosis and treatment of malignant lung lesions can substantially increase survival rates $[6,7]$.

Despite these advantages, the diagnostic yield of all guided bronchoscopy systems has historically been limited by a phenomenon called computed tomography (CT)-to-body divergence [8]. This effect refers to a mismatch between the preprocedure CT-based virtual map used to plan the navigation route to the lung lesion and the actual dynamic lung anatomy during bronchoscopy. This divergence can be caused by a number of factors [8] and has been addressed, in part, by advanced bronchoscopy systems that provide real-time visualization and/ or positional correction during the bronchoscopy procedure [9-18]. However, anesthesia-induced atelectasis remains a challenge that can obscure lesion visibility and cause inaccurate localization, even with intraprocedural positional correction.

While it has long been known that nearly all patients experience atelectasis within minutes after general anesthesia induction [19-23], atelectasis during bronchoscopy and its impact on outcomes has been underappreciated until recently. Because atelectasis is not visible on standard fluoroscopy $[24,25]$, its prevalence during bronchoscopy was not realized until the use of $\mathrm{CBCT}$ became more common. Using $\mathrm{CBCT}$, Casal et al. were the first to report atelectasis in dependent areas in $40 \%$ of peripheral lung biopsies, completely obscuring the target in $20 \%$ of cases [24]. Avasarala et al. reported atelectasis in $75 \%$ of patients, obscuring the lesion in $38 \%$ [26]. In the first prospective study specifically designed to assess atelectasis during bronchoscopy (I-LOCATE), Sagar et al. reported that $89 \%$ of patients had atelectasis in at least one bronchial segment with a prevalence greater than $50 \%$ in dependent lower lobe segments. Atelectasis was observed within 30 min of anesthesia induction. Increased body mass index and time to atelectasis assessment were significant predictors of atelectasis risk [25]. General anesthesia was used with a laryngeal mask airway (LMA) and neuromuscular blocking agents, and most patients were ventilated with $100 \%$ fraction of inspired oxygen $\left(\mathrm{FiO}_{2}\right)$ and zero to minimal PEEP [25].

Atelectasis can have a significant impact on the success of guided bronchoscopy. Atelectasis decreases the visibility of lung lesions on imaging and changes the conformation of the airways (Fig. 1), exacerbating any mismatch between the virtual lung map and the patient's anatomy. Atelectasis reduces the distance between the lesion and the pleura, increasing the risk of pneumothorax from
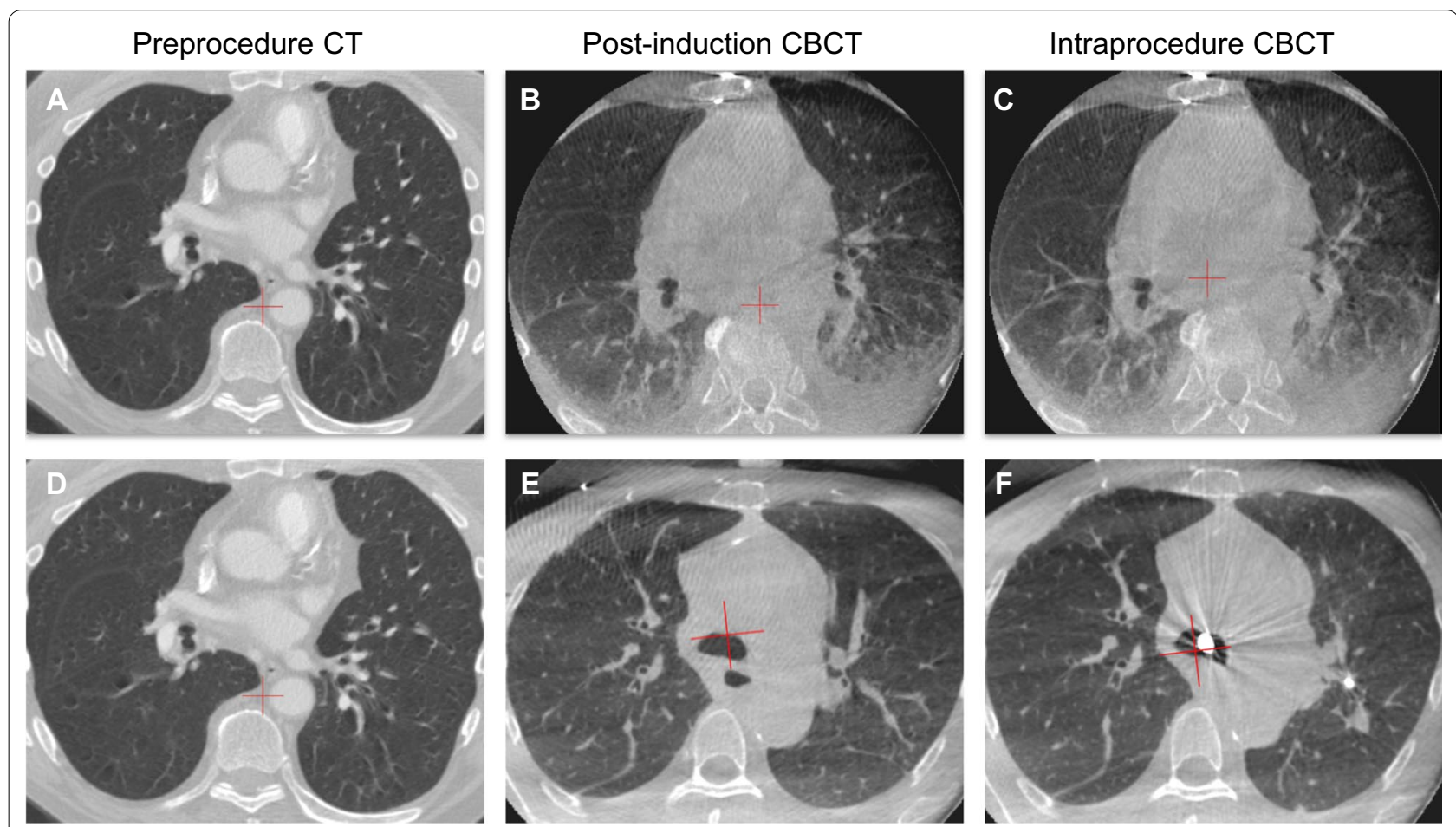

Fig. 1 A-C Computed tomography (CT) and cone-beam CT (CBCT) scans during image-guided bronchoscopy without an optimized ventilation protocol. Significant atelectasis and ghosting artifact were observed. D-F CT and CBCT scans from a different patient using a ventilation protocol designed to prevent atelectasis 
instrumentation. Because of its increased density compared to aerated lung, atelectasis can also create false positive findings on radial EBUS that mimic a lung lesion [25]. The result is inaccurate localization and reduced diagnostic yield [27]. Significant changes in inflation pressure are required to open atelectatic lung units. Because recruitment maneuvers to temporarily increase PEEP and tidal volumes often fail to resolve the atelectasis once it has appeared [24], it is important to prevent atelectasis from occurring and maintain optimal conditions throughout the bronchoscopic navigation and biopsy procedures.

\section{Literature review}

A systematic literature search of MEDLINE and Embase was conducted for papers evaluating the impact of anesthesia methods on outcomes during advanced peripheral bronchoscopy (ENB, radial EBUS, VBN, CBCT, augmented fluoroscopy, robotic bronchoscopy). Detailed methods are provided in Additional file 1: Figure S1, Additional file 1: Table S1 and Additional file 1: Table S2. After abstract and full-text review, there were 11 papers (8 original studies and 3 meta-analyses) that specifically evaluated the impact of the anesthesia method on atelectasis, safety, or diagnostic yield during advanced bronchoscopy [4, 27-36]. Bowling et al. compared ENB using general anesthesia versus intravenous sedation and observed diagnostic yields of $70 \%$ and $78 \%$, respectively $(P=\mathrm{NS})$ [29]. As will be described in greater detail below, Bhadra et al. 2021 observed less atelectasis and a trend toward greater diagnostic yield using an optimized ventilation protocol compared to conventional ventilation [28]. Minami et al. (2017) reported that fentanyl and midazolam sedation was useful for peripheral bronchoscopy [32]. Webb et al. (2020) reported that jet ventilation was associated with reduced target displacement and increased diagnostic yield compared to traditional ventilation during ENB [35]. General anesthesia and conscious sedation were used with approximately equal frequency across studies in a 2020 meta-analysis with no significant differences in diagnostic yield [4], consistent with results from the AQuIRE registry [33], a 2015 meta-analysis [36], and the NAVIGATE study [30]. One 2014 meta-analysis observed significantly higher diagnostic yield with general anesthesia use compared to conscious sedation [31] and the NAVIGATE study found a significant effect of general anesthesia versus moderate sedation on complication rates [34]. Finally, Tanner et al. found no significant difference in diagnostic yield using moderate versus deep sedation using standard bronchoscopy or radial EBUS [27].

However, with one exception [28], the studies described above used navigation bronchoscopy systems without intraprocedural advanced imaging. Newer systems such as tomosynthesis-based fluoroscopic navigation [10] and CT augmented fluoroscopy [28] require a breath hold to minimize lung movement during intraprocedural imaging and location adjustments. Furthermore, CT-to-body divergence and atelectasis are likely strong contributors to the sub-optimal diagnostic yield seen with earlier generations of navigation bronchoscopy technology in large multicenter studies [30].

\section{Ventilation recommendations}

The following is a recommended protocol for ventilation during advanced guided bronchoscopic biopsy (Table 1). These recommendations were derived from the anesthesia literature regarding the principles of minimizing atelectasis during positive pressure ventilation [37-42], the authors' published work [28], and the authors' combined experience of over 1000 CBCT navigation cases assessing the presences of atelectasis and CT-to-body divergence. While the use of higher PEEP and lower $\mathrm{FiO}_{2}$ during induction may contradict traditional anesthesiology practices, they are important for guided bronchoscopy where the success of the biopsy is highly dependent upon stabilizing dependent areas of the lung during intraprocedural imaging, particularly in obese patients and those with posterior or lower lobe lesions $[43,44]$. These techniques will help to reduce movement and minimize atelectasis, thereby increasing the chances of a successful biopsy and potentially accelerating the time to treatment by reducing the need for repeat biopsy procedures. These methods should be used at the discretion of the anesthesiologist and the proceduralist based on the individual risk factors of each patient. Standard monitoring procedures should be followed according to the American Society of Anesthesiologists (ASA) recommendations.

\section{Preprocedure incentive spirometry}

Preprocedure incentive spirometry has been recommended to recruit lung volume and prevent atelectasis [45]. The incentive spirometry maximum value will also allow the anesthesiologist to anticipate any risks of the higher intraprocedural tidal volumes recommended below. This is especially important for patients at particular risk of intraprocedural atelectasis, such as obese patients or those with lesions in the dependent areas of the lung.

\section{Preoxygenation}

The benefits of pre-oxygenation prior to anesthetic induction and tracheal intubation are widely accepted. However, nitrogen washout during pre-oxygenation promotes loss of gas from the lung to the bloodstream, 
Table 1 Anesthesia for advanced guided bronchoscopy

\begin{tabular}{|c|c|c|c|}
\hline & Step & Considerations & Recommendations \\
\hline 1 & Preprocedure & $\begin{array}{l}\text { Recruit lung volume, assess tolerance to higher PEEP, and } \\
\text { prevent atelectasis }\end{array}$ & Perform incentive spirometry \\
\hline 2 & Preoxygenation & Avoid absorption atelectasis & Modest $\mathrm{FiO}_{2}(0.6$ to 0.8$)$ as tolerated \\
\hline 3 & Anesthesia type & Need for a completely motionless patient & TIVA with propofol and muscle paralysis \\
\hline \multirow[t]{2}{*}{4} & Intubation & $\begin{array}{l}\text { Enable gas passage past the bronchoscope with the least } \\
\text { increase in circuit pressure }\end{array}$ & $\begin{array}{l}\text { Use a larger endotracheal tube (usually } \geq 8.5 \text {, but as guided } \\
\text { by patient anatomy) }\end{array}$ \\
\hline & & $\begin{array}{l}\text { Minimize atelectasis by avoiding traditional rapid-sequence } \\
\text { intubation (i.e., avoid } \mathrm{FiO}_{2} \text { of } 1.0 \text { and Suxamethonium) }\end{array}$ & $\begin{array}{l}\text { Perform an expeditious intubation using non-depolarizing } \\
\text { muscle relaxants }\end{array}$ \\
\hline \multirow[t]{4}{*}{5} & Post-intubation & Reverse any induction-related atelectasis and assess hemo- & Conduct up to 4 recruitment maneuvers as tolerated \\
\hline & & dynamic stability during higher PEEP & Maintain $\mathrm{FiO}_{2}$ at the lowest tolerable level \\
\hline & & Maintain optimal lung inflation & $\begin{array}{l}\text { PEEP of up to } 10-12 \mathrm{~cm} \mathrm{H}_{2} \mathrm{O} \text { for upper lobe biopsies, con- } \\
\text { sider higher PEEP for lower lobe lesions or obese patients }\end{array}$ \\
\hline & & & An increase in tidal volumes may be considered \\
\hline \multirow[t]{3}{*}{6} & Breath-hold: timing & Reduce motion artifact & Breath-hold at peak inspiration (end of a normal tidal breath) \\
\hline & Breath-hold: pressure & $\begin{array}{l}\text { Maintain a constant circuit pressure and PEEP and reduce } \\
\text { diaphragmatic movement }\end{array}$ & $\begin{array}{l}\text { Manually adjust APL valve to maintain circuit pressure at } \\
\text { desired PEEP level }\end{array}$ \\
\hline & Breath-hold: duration & $\begin{array}{l}\text { To minimize lung movement during imaging, allow time } \\
\text { for pressure to equilibrate }\end{array}$ & $\begin{array}{l}\text { Maintain breath-hold for 5-10 s before beginning imaging } \\
\text { sweep }\end{array}$ \\
\hline 7 & Biopsy & Ensure consistent settings between imaging and biopsy & Maintain settings at the same levels as Step 6 \\
\hline 8 & Post-procedure & Exclude pneumothorax and assess any residual atelectasis & $\begin{array}{l}\text { Routine reversal and post-procedure methods. Perform } \\
\text { chest X-ray }\end{array}$ \\
\hline
\end{tabular}

$A P L$ adjustable pressure-limiting valve, $\mathrm{FiO}_{2}$ fraction of inspired oxygen, $P E E P$ positive end-expiratory pressure, TIVA total intravenous anesthesia

resulting in alveolar collapse and absorption atelectasis. Gas absorption accelerates airway collapse by a combination of decreased functional residual capacity and compression atelectasis [46]. Therefore, the use of $100 \%$ oxygen during induction and anesthesia maintenance is a major cause of atelectasis [41]. Given the risk of absorption atelectasis and lung injury, the lowest tolerable $\mathrm{FiO}_{2}$ is recommended for preoxygenation [46] as guided by oxygen saturation. Edmark et al. demonstrated that in most patients, an $\mathrm{FiO}_{2}$ of 0.8 to 0.6 was associated with little to no atelectasis compared to an $\mathrm{FiO}_{2}$ of 1.0 [37]. Inspired oxygen content should also be kept as low as tolerated during the procedure to minimize absorption atelectasis.

If it is not possible to decrease the $\mathrm{FiO}_{2}$ to less than 1.0 during pre-induction, the $\mathrm{FiO}_{2}$ should be kept at the lowest tolerable level immediately after the endotracheal tube (ETT) cuff is inflated, and recruitment maneuvers should be performed with the lowered $\mathrm{FiO}_{2}$. This lowered $\mathrm{FiO}_{2}$ should be maintained for the remainder of the procedure, including during the biopsy, as the patient's oxygen saturation permits.

\section{Anesthesia type and intubation}

The need for a completely motionless patient during guided bronchoscopy necessitates general anesthesia, paralysis, and intubation. The largest ETT size feasible based on patient anatomy will enable gas passage around the bronchoscope (which ranges in diameter from 5.9 to $6.3 \mathrm{~mm}$ ) with the least increase in circuit pressure [47]. An ETT is preferred over an LMA to accommodate higher airway pressures (larger tidal volumes and increased PEEP). LMA use may increase the risk of gastric insufflation with higher pressures. This in turn may increase the risk for aspiration, although the risk remains low based on previous studies [48]. Following bronchoscopy, the ETT can be exchanged for an LMA to facilitate complete EBUS staging if desired. LMA may be feasible in smaller patients (e.g., $<80 \mathrm{~kg}$ ) provided that a good seal can be obtained whilst providing the recommended tidal volumes and PEEP. Jet ventilation has also been used to minimize motion and CT-to-body divergence [35, 49], but may be limited by availability and expertise.

General anesthesia using total intravenous anesthesia (TIVA) with propofol and muscle paralysis is optimal [47]. Lengthy intubation times may increase the risk of atelectasis, thus, avoid "traditional" rapid-sequence intubation (i.e., avoid the use of $\mathrm{FiO}_{2}$ of 1.0). Instead, perform an expeditious intubation using non-depolarizing muscle relaxants rather than suxamethonium. Application of PEEP throughout induction has also been shown to prevent atelectasis [42]. Whilst the use of volatile anesthetics is not contraindicated, repeated opening of the circuit for the passage of the scope poses additional challenges to maintaining the depth of anesthesia and volatile gas pollution in the operating room. 


\section{Recruitment maneuvers}

Reducing the $\mathrm{FiO}_{2}$ to the lowest tolerable percentage after intubation will improve airway visualization. Unless contraindicated (e.g., due to acute respiratory distress syndrome, ventilator-induced lung injury, recent segmentectomy, structural lung disease, or surgery), conduct up to four recruitment maneuvers immediately after intubation [46] to reverse any intubation atelectasis and to assess hemodynamic stability during higher PEEP (Fig. 2). This is especially important if intubation was prolonged due to a difficult intubation. Hemodynamic instability may preclude the use of traditional recruitment maneuvers, such as the use of $40 \mathrm{~cm} \mathrm{H}_{2} \mathrm{O}$ for $40 \mathrm{~s}$ (the ' 40 for 40 hold'). Judicious use of PEEP from the preinduction phase and throughout the procedure is recommended [21, 38, 42], but may vary based on the patient's hemodynamic tolerance. Maintain higher PEEP with the lowest tolerable $\mathrm{FiO}_{2}$ as guided by oxygen saturation. PEEP of up to $10-12 \mathrm{~cm} \mathrm{H}_{2} \mathrm{O}$ may be beneficial for upper lobe biopsies, and even higher PEEP may be required for lower lobe biopsies (particularly in obese patients) due to the lower functional residual capacity and increased risk of atelectasis in the dependent areas. An increase in tidal volumes may be considered if tolerated.

Use of higher PEEP and tidal volumes will help maintain optimal lung inflation [44]. However, use of these higher settings should be guided by what the patient is able to tolerate hemodynamically, as demonstrated in the post-intubation recruitment maneuvers. This is especially important in obese patients or those with structural lung disease who may be at increased risk of barotrauma or volutrauma. Also, the risk of barotrauma is minimized when the sheer stress of repeated alveolar re-expansion is avoided, and the alveoli are held on a favorable part of the compliance curve (Fig. 3). While intuitively contradictory to the classical teaching of low tidal volume to prevent barotrauma, airway visualization, accurate lesion localization, and successful biopsy require these preprocedural conditions to be replicated as closely as possible.

\section{Breath-hold for image acquisition Timing the breath-hold}

A breath-hold is required to reduce motion artifact and provide clearer, more accurate images during certain image-guided bronchoscopy procedures (e.g., fluoroscopic navigation, $\mathrm{CBCT}$ ). These procedures require a complete lack of movement beyond cardiac pulsations; therefore, a carefully timed breath-hold is essential. The breath-hold should be performed at peak inspiration, not at end-expiration. This does not require a vital capacity maneuver, rather, it should occur at the end of a normal inspiratory breath (Fig. 4).

\section{Maintaining a constant circuit pressure and PEEP}

The breath-hold may be achieved by either the anesthesia machine's automatic feature or manually. The automatic feature sets and maintains the appropriate pressure using the Vital Capacity Hold Function. In the manual method, the breath-hold is initiated by switching the ventilator to manual mode. Adjusting the adjustable pressure-limiting (APL) valve during breath hold maneuvers is required to maintain a constant circuit pressure and PEEP and reduce diaphragmatic movement once a leak is created in the circuit with the passage of the bronchoscope. The

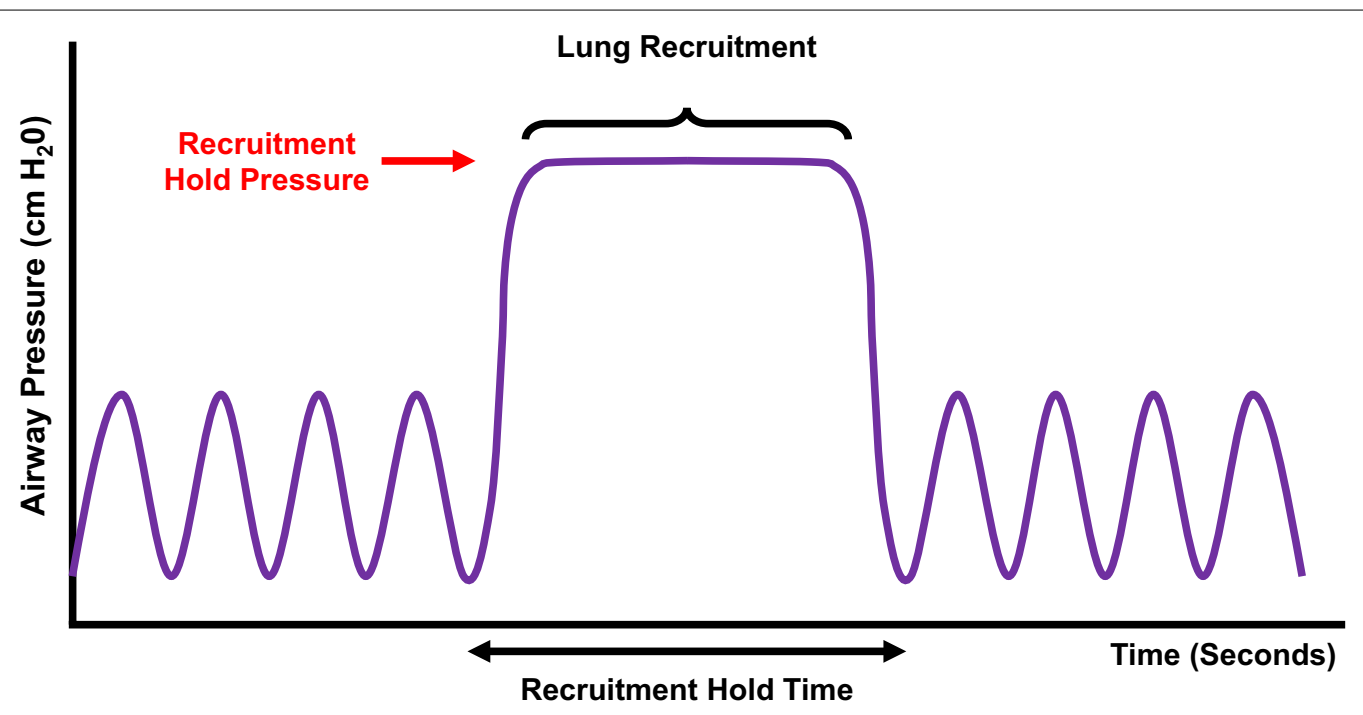

Fig. 2 Recruitment maneuvers after intubation may reverse any induction-related atelectasis and assess hemodynamic stability during higher PEEP. Hemodynamic instability may limit use of the traditional ' 40 for 40 ' hold. Higher PEEP for shorter duration may be considered 
A

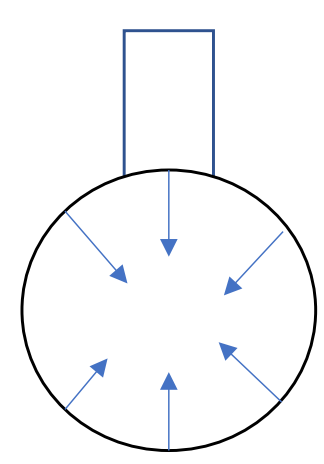

C
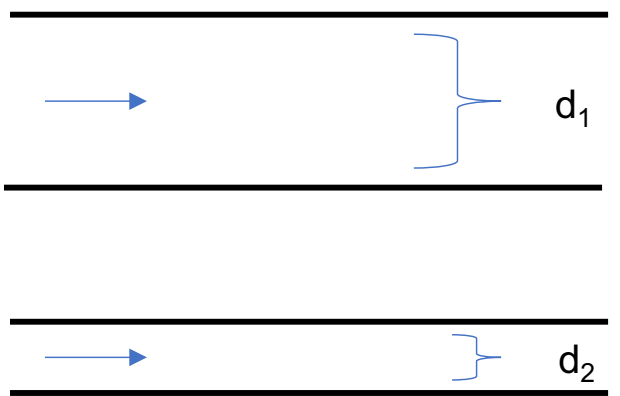

B
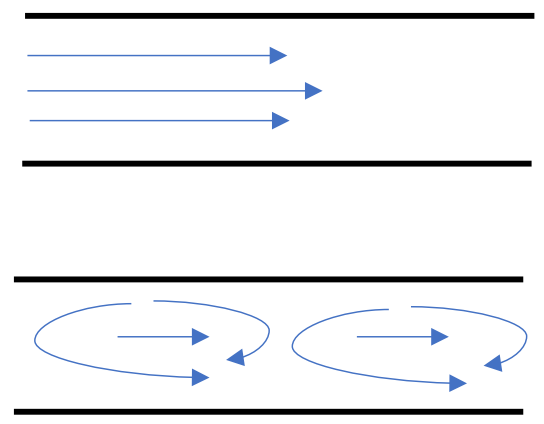

D

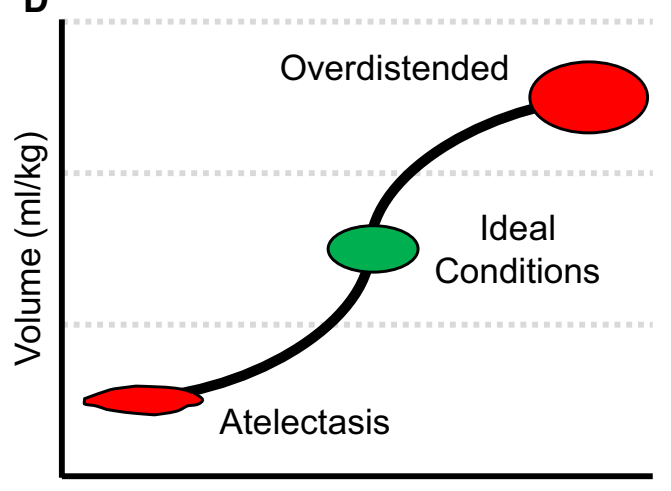

Pressure

Fig. 3 Mechanisms leading to atelectasis during bronchoscopy and increased airway resistance. A Surface tension caused by water molecules leads to attraction. B Once the airflow is disrupted in atelectatic lung units, turbulent airflow leads to increased airway resistance. C As the bronchi decrease in diameter, there is a substantial increase in airways resistance. D Compliance curve for alveoli. As the lung deflates, resistance increases and gas flow through the airways decreases. Atelectatic lung units have poor compliance and require significant changes in inflation pressure to result in minor changes in volume

measured circuit pressure reflects intrapulmonary pressure (PEEP) and is a balance between fresh gas inflow and leak from the circuit plus outflow via the APL. Therefore, it's preferable to focus on the circuit pressure reading (shown either on the digital screen or on the circuit pressure manometer) and adjust the APL manually to maintain this pressure at the designated PEEP, rather than on an absolute APL number. Vigilance is required to ensure that there is a balance of fresh gas inflow to circuit leak. These measures will minimize motion artifact of the diaphragm during imaging which can cause blurring and thereby hinder image interpretation by the clinician.

\section{Maintaining the breath-hold}

The breath-hold should be maintained for a sufficient time to allow pressures to plateau and equilibrate throughout the bronchial tree prior to beginning intraprocedural imaging (Fig. 5). The proceduralist should wait 5-10 s before beginning the imaging sweep after the anesthesiologist initiates the breath-hold (once PEEP is stabilized as measured by constant circuit pressure). This gives time for the intrapulmonary pressures to equilibrate before the imaging sweep begins. These measures will remove motion artifact, provide clearer images, and allow for more accurate localization of the lung lesion. By maintaining high PEEP, the bronchial tree is also optimally dilated. The imaging sweep will add additional time to the breath-hold (up to $30 \mathrm{~s}$, depending on the system used). Therefore, it is important to be aware of the potential for hemodynamic changes due to prolonged breathhold during the equilibration and imaging sweep.

\section{Biopsy procedure}

The recommended anesthesia settings described above should be maintained throughout the biopsy procedure to ensure that lung volumes are consistent with those present during intraprocedural imaging. Any changes during the biopsy procedure would necessitate a repeat of both the breath-hold and the imaging sweep. 


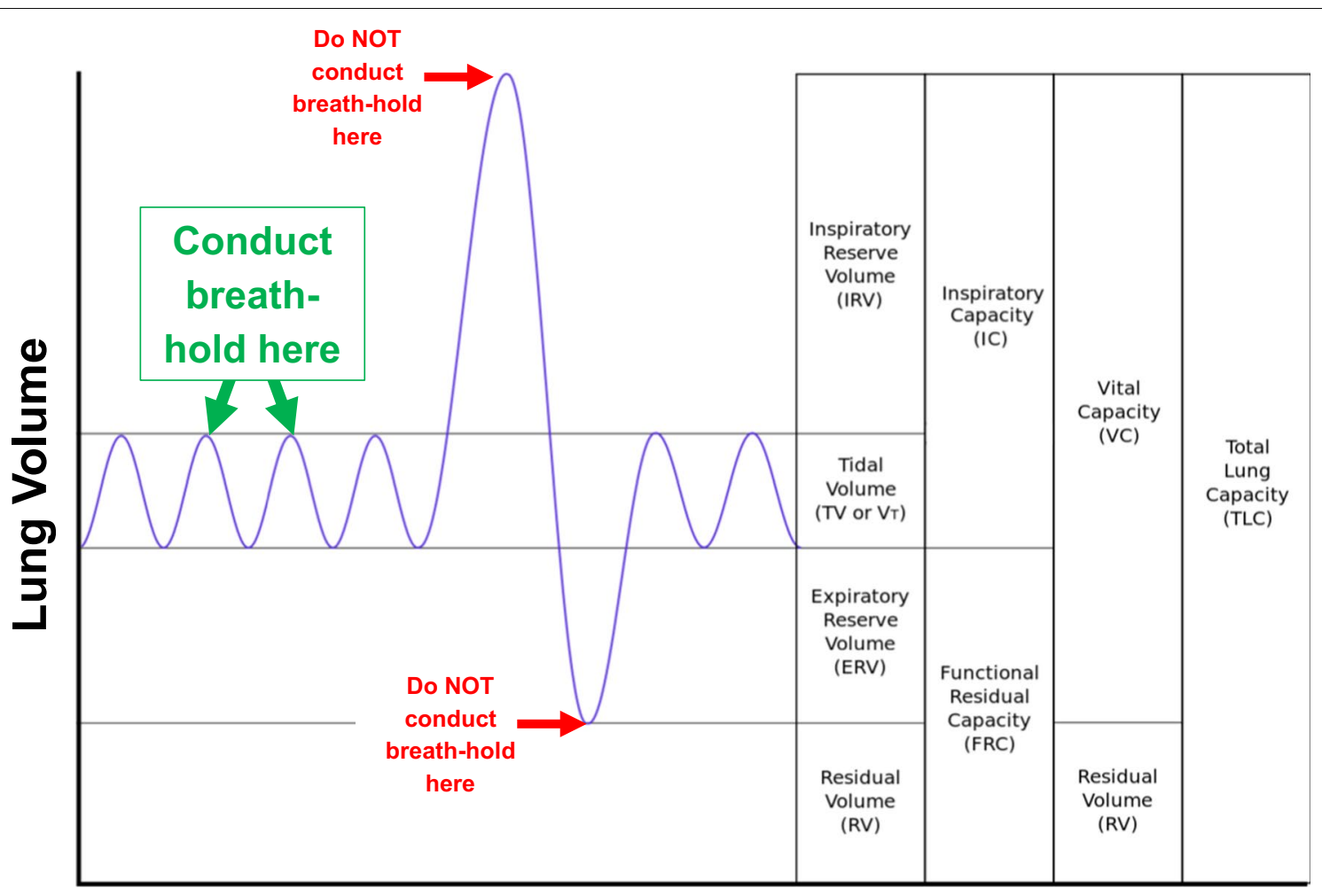

Time

Fig. 4 A breath-hold is required to reduce motion artifact during intraprocedural imaging (e.g., CBCT, digital tomosynthesis). The breath-hold should be performed at peak inspiration, not at end-expiration. This does not require a vital capacity maneuver but should occur at the end of a normal tidal breath. Adapted from Kapwatt at English Wikipedia (https://commons.wikimedia.org/w/index.php?curid=74891988) and used with permission under the terms of Creative Commons License CC BY-SA 3.0 ()

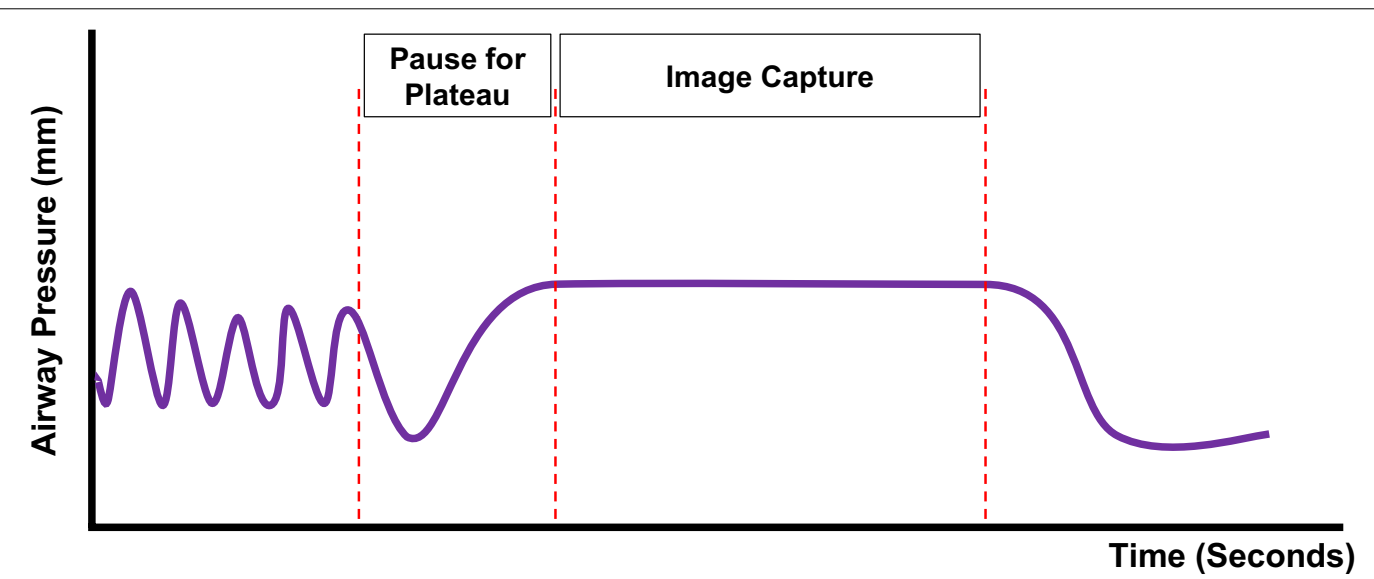

Fig. 5 To prevent motion artifact, maintain the breath-hold until pressures plateau before beginning the imaging sweep (5-10 s). Be aware of the potential for hemodynamic changes due to prolonged breath-hold

\section{Post-procedure}

Routine reversal and standard assessment of fitness for extubation should be employed, as should suctioning of secretions. Standard post-procedural discharge criteria should be employed, and a postprocedure chest $\mathrm{x}$-ray is recommended to exclude complications such as pneumothorax. Patients should be completely reversed from neuromuscular paralysis. 


\section{Clinical evidence}

The proposed ventilation protocol has been evaluated in a retrospective, single-center study of subjects with peripheral lung lesions $<30 \mathrm{~mm}$ undergoing CBCT with augmented fluoroscopy [28]. In two non-randomized, consecutively enrolled groups ( 25 subjects per group), a conventional, non-standardized ventilation protocol (typically $100 \% \mathrm{FiO}_{2}$ and PEEP set to 0 or $5 \mathrm{~cm} \mathrm{H}_{2} \mathrm{O}$ ) was compared to the optimized ventilation protocol. Two independent reviewers observed significantly less atelectasis in dependent areas $(P=0.0001)$ and in sublobar or lobar regions $(P=0.01)$ using the optimized protocol. There was also a significantly smaller proportion of lesions obscured by atelectasis using the optimized protocol (8\% Reviewer 1, 4\% Reviewer 2) than the conventional protocol (36\% observed by both reviewers). Furthermore, this significant reduction in atelectasis was accompanied by a higher diagnostic yield, though not statistically significant (70\% conventional versus $92 \%$ optimized, $P=0.08$ ). Pneumothorax not requiring a chest tube was observed in 1 patient following the optimized protocol compared to 0 patients with conventional ventilation $(P=1.0)$. A study of tomosynthesis-based fluoroscopic navigation used a similar ventilation protocol, with intubation under TIVA, neuromuscular blockade, a recruitment maneuver after intubation, minimized oxygen, and $15 \mathrm{~cm} \mathrm{H}_{2} \mathrm{O}$ applied throughout the procedure. A diagnostic yield of $77 \%$ was observed, with a pneumothorax rate of only $2.5 \%(8 / 324)$ [10]. While future randomized studies are necessary to confirm these results, this data suggests that a ventilation protocol optimized for advanced guided bronchoscopy not only reduces atelectasis, but may also increase in diagnostic yield. The randomized VESPA trial is (NCT04311723) currently enrolling and aims to compare a ventilation strategy designed to prevent atelectasis during bronchoscopy compared to conventional mechanical ventilation [50].

\section{Conclusions}

Atelectasis is common during advanced guided bronchoscopic biopsy [25] and can cause CT-to-body divergence [8], interfering with the ability to obtain diagnostic tissue [28]. The anesthesia techniques proposed in this paper are designed to stabilize dependent areas of the lung, reduce atelectasis, minimize motion artifact, and provide more accurate localization during guided bronchoscopy. Future randomized studies are needed to prospectively compare bronchoscopy procedures with and without optimized ventilation strategies.

\section{Abbreviations}

APL: Adjustable pressure-limiting (valve); ASA: American Society of Anesthesiologists; CBCT: Cone-beam computed tomography; CT: Computed tomography; EBUS: Endobronchial ultrasound; ETT: Endotracheal tube; $\mathrm{FiO}_{2}$ : Fraction of inspired oxygen; LMA: Laryngeal mask airway; PEEP: Positive end-expiratory pressure; TIVA: Total intravenous anesthesia.

\section{Supplementary Information}

The online version contains supplementary material available at https://doi. org/10.1186/s12890-021-01584-6.

Additional file 1. Literature search methods.

\section{Acknowledgements}

The authors wish to thank Jennifer S. Mattingley MD and Mike Calcutt of Medtronic for review and guidance.

\section{Authors' contributions}

$M P$ and $K B$ conceived of the paper and developed the ventilation methodology; MP, KB, KL, and SS validated the methodology; KP refined the methodology; MP, KB, and KP wrote sections of the paper and directed the content; all authors provided interpretation and critically revised all drafts of the paper. All authors read and approved the final manuscript.

\section{Funding}

Supported by Medtronic. Medical writing support was provided by Kristin L. Hood PhD of Medtronic (Minneapolis, MA) in accordance with Good Publication Practice (GPP3) guidelines [51]. The authors had final responsibility for the decision to submit for publication and were not paid to write this article by Medtronic or any other agency.

Availability of data and materials

Not applicable.

\section{Declarations}

Ethics approval and consent to participate

Not applicable.

\section{Consent for publication \\ Not applicable.}

\section{Competing interests}

K. Bhadra discloses consulting/speaking payments from Medtronic, Boston Scientific, BodyVision, Auris Health, Inc., Intuitive Surgical, Veracyte, Biodesix, Merit Medical Endotek, and Johnson \& Johnson. K. Lau discloses consulting/ speaking payments from Medtronic, Philips, Intuitive Surgical, and Johnson \& Johnson. K. Phillips is a full-time employee of Medtronic. M. Pritchett discloses speaking, consulting, or research payments from Medtronic, Auris Health, Inc, BodyVision, Intuitive Surgical, Philips, Biodesix, AstraZeneca, Johnson \& Johnson/NeuWave, Actelion, Inivata, and Boston Scientific. S. Skibo discloses speaking/consulting payments from Medtronic, Biodesix, GlaxoSmithKline and AstraZeneca.

\section{Author details}

${ }^{1}$ Chest Center of the Carolinas at First Health, President of the Society for Advanced Bronchoscopy, FirstHealth of the Carolinas and Pinehurst Medical Clinic, 205 Page Road, Pinehurst, NC 28374, USA. ${ }^{2}$ Thoracic Surgery, St. Bartholomew's Hospital, West Smithfield, London EC1A 7BE, UK. ${ }^{3}$ Interventional Thoracic Oncology, Pulmonary Critical Care, Haywood Regional Medical Center (A Duke LifePoint Hospital), 262 Leroy George Drive, Clyde, NC 28721, USA. ${ }^{4}$ Anesthesiologist and Intensivist, Medtronic, 2101 Faraday Avenue, Carlsbad, CA 92008, USA. ${ }^{5}$ Interventional Pulmonology, CHI Memorial Rees Skillern Cancer Institute, 725 Glenwood Dr E-500, Chattanooga, TN 37401, USA.

Received: 1 March 2021 Accepted: 5 July 2021

Published online: 17 July 2021 


\section{References}

1. Cicenia J, Avasarala SK, Gildea TR. Navigational bronchoscopy: a guide through history, current use, and developing technology. J Thorac Dis. 2020;12:3263-71

2. Katsis JM, Rickman OB, Maldonado F, Lentz RJ. Bronchoscopic biopsy of peripheral pulmonary lesions in 2020: a review of existing technologies. J Thorac Dis. 2020;12:3253-62.

3. Heerink WJ, de Bock GH, de Jonge GJ, Groen HJ, Vliegenthart R, Oudkerk M. Complication rates of CT-guided transthoracic lung biopsy: metaanalysis. Eur Radiol. 2017;27:138-48.

4. Folch EE, Labarca G, Ospina-Delgado D, Kheir F, Majid A, Khandhar SJ, et al. Sensitivity and safety of electromagnetic navigation bronchoscopy for lung cancer diagnosis: systematic review and meta-analysis. Chest. 2020;158:1753-69.

5. Rivera MP, Mehta AC, Wahidi MM. Establishing the diagnosis of lung cancer: diagnosis and management of lung cancer, 3rd edition: American College of Chest Physicians evidence-based clinical practice guidelines. Chest. 2013;143:e142S-e165S.

6. Siegel RL, Miller KD, Fuchs HE, Jemal A. Cancer statistics, 2021. CA Cancer J Clin. 2021:71:7-33

7. Henschke CI, Yankelevitz DF, Libby DM, Pasmantier MW, Smith JP, Miettinen OS. Survival of patients with stage I lung cancer detected on CT screening. N Engl J Med. 2006;355:1763-71.

8. Pritchett MA, Bhadra K, Calcutt M, Folch E. Virtual or reality: divergence between preprocedural computed tomography scans and lung anatomy during guided bronchoscopy. J Thorac Dis. 2020;12:1595-611.

9. Katsis J, Roller L, Lester M, Johnson J, Lentz R, Rickman O, et al. High Accuracy of digital tomosynthesis-guided bronchoscopic biopsy confirmed by intraprocedural computed tomography. Respiration. 2021:100:1-8.

10. Katsis J, Roller L, Aboudara M, Pannu J, Chen H, Johnson J, et al. Diagnostic yield of digital tomosynthesis-assisted navigational bronchoscopy for indeterminate lung nodules. J Bronchol Interv Pulmonol. 2021. https:// doi.org/10.1097/LBR.0000000000000766.

11. Pritchett MA, Bhadra K, Mattingley JS. Electromagnetic navigation bronchoscopy with tomosynthesis-based visualization and positional correction: three-dimensional accuracy as confirmed by cone-beam computed tomography. J Bronchol Interv Pulmonol. 2021;28:10-20.

12. Pritchett MA. Prospective analysis of a novel endobronchial augmented fluoroscopic navigation system for diagnosis of peripheral pulmonary lesions. J Bronchol Interv Pulmonol. 2021;28:107-15.

13. Thiboutot J, Lee HJ, Silvestri GA, Chen A, Wahidi MM, Gilbert CR, et al. Study design and rationale: a multicenter, prospective trial of electromagnetic bronchoscopic and electromagnetic transthoracic navigational approaches for the biopsy of peripheral pulmonary nodules (ALL IN ONE Trial). Contemp Clin Trials. 2018;71:88-95.

14. Fielding DIK, Bashirzadeh F, Son JH, Todman M, Chin A, Tan L, et al. First human use of a new robotic-assisted fiber optic sensing navigation system for small peripheral pulmonary nodules. Respiration. 2019;98:142-50.

15. Benn BS, Romero AO, Lum M, Krishna G. Robotic-assisted navigation bronchoscopy as a paradigm shift in peripheral lung access. Lung. 2021;199:177-86.

16. Chen AC, Pastis NJ Jr, Mahajan AK, Khandhar SJ, Simoff MJ, Machuzak MS, et al. Robotic bronchoscopy for peripheral pulmonary lesions: a multicenter pilot and feasibility study (BENEFIT). Chest. 2021;159:845-52.

17. Chaddha U, Kovacs SP, Manley C, Hogarth DK, Cumbo-Nacheli G, Bhavani SV, et al. Robot-assisted bronchoscopy for pulmonary lesion diagnosis: results from the initial multicenter experience. BMC Pulm Med. 2019;19:243.

18. Cicenia J, Bhadra K, Sethi S, Nader DA, Whitten P, Hogarth DK. Augmented fluoroscopy: a new and novel navigation platform for peripheral bronchoscopy. J Bronchol Interv Pulmonol. 2021;28:116-23.

19. Gamsu G, Singer MM, Vincent HH, Berry S, Nadel JA. Postoperative impairment of mucous transport in the lung. Am Rev Respir Dis. 1976:114:673-9.

20. Strandberg A, Tokics L, Brismar B, Lundquist H, Hedenstierna G. Atelectasis during anaesthesia and in the postoperative period. Acta Anaesthesiol Scand. 1986;30:154-8.

21. Brismar B, Hedenstierna G, Lundquist H, Strandberg A, Svensson L, Tokics L. Pulmonary densities during anesthesia with muscular relaxation-a proposal of atelectasis. Anesthesiology. 1985;62:422-8.
22. Lundquist $H$, Hedenstierna $G$, Strandberg A, Tokics L, Brismar B. CT-assessment of dependent lung densities in man during general anaesthesia. Acta Radiol. 1995:36:626-32.

23. Duggan M, Kavanagh BP. Pulmonary atelectasis: a pathogenic perioperative entity. Anesthesiology. 2005;102:838-54.

24. Casal RF, Sarkiss M, Jones AK, Stewart J, Tam A, Grosu HB, et al. Cone beam computed tomography-guided thin/ultrathin bronchoscopy for diagnosis of peripheral lung nodules: a prospective pilot study. J Thorac Dis. 2018;10:6950-9.

25. Sagar AS, Sabath BF, Eapen GA, Song J, Marcoux M, Sarkiss M, et al. Incidence and location of atelectasis developed during bronchoscopy under general anesthesia: the I-LOCATE trial. Chest. 2020;158:2658-66.

26. Avasarala SK, Machuzak MS, Gildea TR. Multidimensional precision: hybrid mobile 2D/3D C-arm assisted biopsy of peripheral lung nodules. J Bronchol Interv Pulmonol. 2020;27:153-5.

27. Tanner NT, Yarmus L, Chen A, Wang Memoli J, Mehta HJ, Pastis NJ, et al. Standard bronchoscopy with fluoroscopy versus thin bronchoscopy and radial endobronchial ultrasound for biopsy of pulmonary lesions: a multicenter, prospective. Randomized Trial Chest. 2018;154:1035-43.

28. Bhadra K, Setser RM, Condra W, Pritchett MA. Lung navigation ventilation protocol to optimize biopsy of peripheral lung lesions. J Bronchol Interv Pulmonol. 2021. https://doi.org/10.1097/LBR.0000000000 000756.

29. Bowling MR, Kohan MW, Walker P, Efird J, Ben OS. The effect of general anesthesia versus intravenous sedation on diagnostic yield and success in electromagnetic navigation bronchoscopy. J Bronchol Interv Pulmonol. 2015:22:5-13

30. Folch EE, Pritchett MA, Nead MA, Bowling MR, Murgu SD, Krimsky WS, et al. Electromagnetic navigation bronchoscopy for peripheral pulmonary lesions: one-year results of the prospective, multicenter NAVIGATE study. J Thorac Oncol. 2019;14:445-58.

31. Gex G, Pralong JA, Combescure C, Seijo L, Rochat T, Soccal PM. Diagnostic yield and safety of electromagnetic navigation bronchoscopy for lung nodules: a systematic review and meta-analysis. Respiration. 2014;87:165-76.

32. Minami D, Nakasuka T, Ando C, Iwamoto MdY, Sato K, Fujiwara K, et al. Bronchoscopic diagnosis of peripheral pulmonary lung cancer employing sedation with fentanyl and midazolam. Respir Investig. 2017;55:314-7.

33. Ost DE, Ernst A, Lei X, Kovitz KL, Benzaquen S, Diaz-Mendoza J, et al. Diagnostic yield and complications of bronchoscopy for peripheral lung lesions: results of the AQuIRE registry. Am J Respir Crit Care Med. 2016;193:68-77.

34. Towe CW, Nead MA, Rickman OB, Folch EE, Khandhar SJ, Perry Y, et al. Safety of electromagnetic navigation bronchoscopy in patients With COPD: results From the NAVIGATE study. J Bronchol Interv Pulmonol. 2019:26:33-40.

35. Webb TN, Bonta DV, Masters R, Parks C, Bechara R. Jet ventilation decreases target motion and increases yield of ENB especially in lesions with negative bronchus sign. J Bronchol Interv Pulmonol. 2020;27:14-21.

36. Zhang W, Chen S, Dong X, Lei P. Meta-analysis of the diagnostic yield and safety of electromagnetic navigation bronchoscopy for lung nodules. J Thorac Dis. 2015:7:799-809.

37. Edmark L, Kostova-Aherdan K, Enlund M, Hedenstierna G. Optimal oxygen concentration during induction of general anesthesia. Anesthesiology. 2003;98:28-33

38. Coussa M, Proietti S, Schnyder P, Frascarolo P, Suter M, Spahn DR, et al. Prevention of atelectasis formation during the induction of general anesthesia in morbidly obese patients. Anesth Analg. 2004;98:1491-5.

39. Tusman G, Bohm SH, Warner DO, Sprung J. Atelectasis and perioperative pulmonary complications in high-risk patients. Curr Opin Anaesthesiol. 2012;25:1-10.

40. Hemmes SN, Serpa Neto A, Schultz MJ. Intraoperative ventilatory strategies to prevent postoperative pulmonary complications: a meta-analysis. Curr Opin Anaesthesiol. 2013;26:126-33.

41. Rothen HU, Sporre B, Engberg G, Wegenius G, Reber A, Hedenstierna $\mathrm{G}$. Prevention of atelectasis during general anaesthesia. Lancet. 1995;345:1387-91.

42. Rusca M, Proietti S, Schnyder P, Frascarolo P, Hedenstierna G, Spahn DR, et al. Prevention of atelectasis formation during induction of general anesthesia. Anesth Analg. 2003:97:1835-9. 
43. Eichenberger A, Proietti S, Wicky S, Frascarolo P, Suter M, Spahn DR, et al Morbid obesity and postoperative pulmonary atelectasis: an underestimated problem. Anesth Analg. 2002;95:1788-92.

44. Magnusson L, Spahn DR. New concepts of atelectasis during general anaesthesia. Br J Anaesth. 2003;91:61-72.

45. Eltorai AEM, Baird GL, Eltorai AS, Pangborn J, Antoci V Jr, Cullen HA, et al. Perspectives on incentive spirometry utility and patient protocols. Respir Care. 2018;63:519-31.

46. Nimmagadda U, Salem MR, Crystal GJ. Preoxygenation: physiologic basis, benefits, and potential risks. Anesth Analg. 2017;124:507-17.

47. Galway U, Zura A, Khanna S, Wang M, Turan A, Ruetzler K. Anesthetic considerations for bronchoscopic procedures: a narrative review based on the Cleveland Clinic experience. J Thorac Dis. 2019;11:3156-70.

48. Brimacombe J. The advantages of the LMA over the tracheal tube or facemask: a meta-analysis. Can J Anaesth. 1995;42:1017-23.

49. Hohenforst-Schmidt W, Zarogoulidis P, Huang H, Man Y-G, Laskou S, Koulouris C, et al. A new and safe mode of ventilation for interventional pulmonary medicine: the ease of nasal superimposed high frequency jet ventilation. J Cancer. 2018;9:816-33.

50. Ventilatory strategy for the prevention of atelectasis during bronchoscopy under general anesthesia, VESPA trial (NCT04311723). ClinicalTrials. gov website. 2020. https://clinicaltrials.gov/ct2/show/NCT04311723. Accessed 31 May 2021

51. Battisti WP, Wager E, Baltzer L, Bridges D, Cairns A, Carswell Cl, et al. Good publication practice for communicating company-sponsored medical research: GPP3. Ann Intern Med. 2015;163:461-4.

\section{Publisher's Note}

Springer Nature remains neutral with regard to jurisdictional claims in published maps and institutional affiliations.
Ready to submit your research? Choose BMC and benefit from:

- fast, convenient online submission

- thorough peer review by experienced researchers in your field

- rapid publication on acceptance

- support for research data, including large and complex data types

- gold Open Access which fosters wider collaboration and increased citations

- maximum visibility for your research: over $100 \mathrm{M}$ website views per year

At BMC, research is always in progress.

Learn more biomedcentral.com/submissions 